Doug Geisler, Eva K. Grebel, and Dante Minniti, eds.

\title{
Ages and Metallicities of Globular Clusters in NGC 4472
}

\author{
M.A. Beasley, R.M. Sharples \\ University of Durham, Department of Physics, South Road, Durham, \\ DH1 $3 L E, U K$
}

\section{T.J. Bridges}

Anglo-Australian Observatory, P.O. Box 296, Epping, NSW, 2121, Australia

\section{D.A. Hanes}

Department of Physics, Queen's University, Kingston, ON K7L 3N6, Canada

\section{S.E. Zepf}

Dept. of Astronomy, Yale University, New Haven, CT 06520, USA

\section{K.M. Ashman}

Department of Physics and Astronomy, University of Kansas, Lawrence $K S 66045, U S A$

D. Geisler

Departamento de Fisica, Grupo de Astronomia, Universidad de Concepción, Casilla 160-C, Concepción, Chile

\begin{abstract}
We have derived ages and metallicities from co-added spectra of 131 globular clusters (GCs) associated with the giant Virgo elliptical NGC 4472. ¿From their metal-line indices, we find that our sample of GCs span a metallicity range of approximately $-1.6 \leq[\mathrm{Fe} / \mathrm{H}] \leq 0$ dex. The SSP models of Worthey (1994; W94) predict that the metal-poor population of GCs has an age of $14.5 \pm 4 \mathrm{Gyr}$ and that the metal-rich population is $13.8 \pm 6 \mathrm{Gyr}$ old, whilst the models of Kurth, Fritze-v. Alvensleben \& Fricke (1999; KFF99) predict ages of $6.0 \pm 2$ and $8.0 \pm$ 5 respectively. We conclude that, within the uncertainties, the GCs are old and coeval, and that the bimodality seen in the broadband colours primarily reflects metallicity and not age differences.
\end{abstract}

\section{Introduction}

We have undertaken a project to obtain a large number of spectra of globular clusters (GCs) associated with the giant Virgo elliptical NGC 4472, in an effort to understand their ages, metallicities and dynamics. 


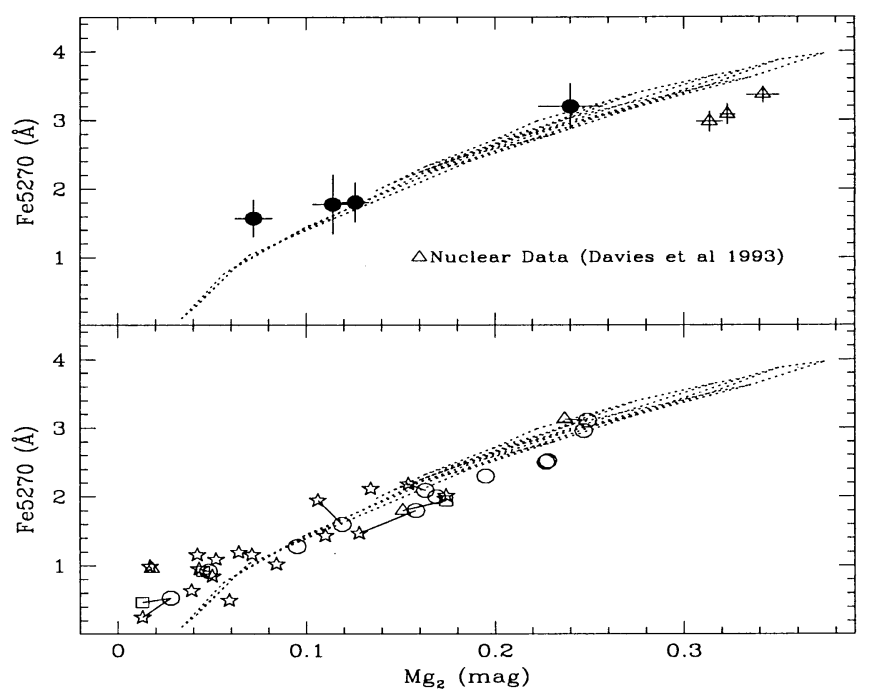

Figure 1. Globular clusters in the Fe $5270-\mathrm{Mg}_{2}$ plane. Dotted lines represent W94 models. Top panel: NGC 4472 GCs are represented by filled circles, galaxy data (Davies et al. 1993) are open triangles. Bottom panel: Various data for Milky Way GCs, lines connect the same GCs between different datasets.

We have now acquired a dataset comprising of 144 GC spectra, and their kinematics are analysed in Zepf et al. (2000) (see also T. Bridges - this volume). Here we present results of the line-strength analysis (see Beasley et al. 2000).

\section{Observations and Analysis}

Spectroscopic targets were selected from the catalogue of Geisler, Lee \& Kim (1996; GLK96), identified from $C, T_{1}$ KPNO 4-m prime focus images of NGC 4472. Multi-slit spectra in the wavelength range 3800-6500 $\AA$ were obtained using LDSS++ on the WHT 4.2-m telescope and MOS at the CFHT 3.9-m telescope. To increase the $\mathrm{S} / \mathrm{N}$ of the GCs for line-strength analysis, we have separated the spectra into bins by their $C-T_{1}$ colours, and combine then on this basis.

\section{Results}

In Fig. 1, we show our NGC 4472 data in the Fe5270-Mg2 plane, compared to the central galaxy values (top panel) and a sample of Milky Way GCs (bottom panel). The NGC 4472 GCs show very similar behaviour to the Milky Way GCs, but are clearly not as metal-rich or as magnesium enhanced as NGC 4472 itself.

We compare our data to the SSP models of W94 and KFF99 in Fig. 2. Using the W94 models, we obtain ages of $14.5 \pm 4$ and $13.8 \pm 6$ Gyr for the 

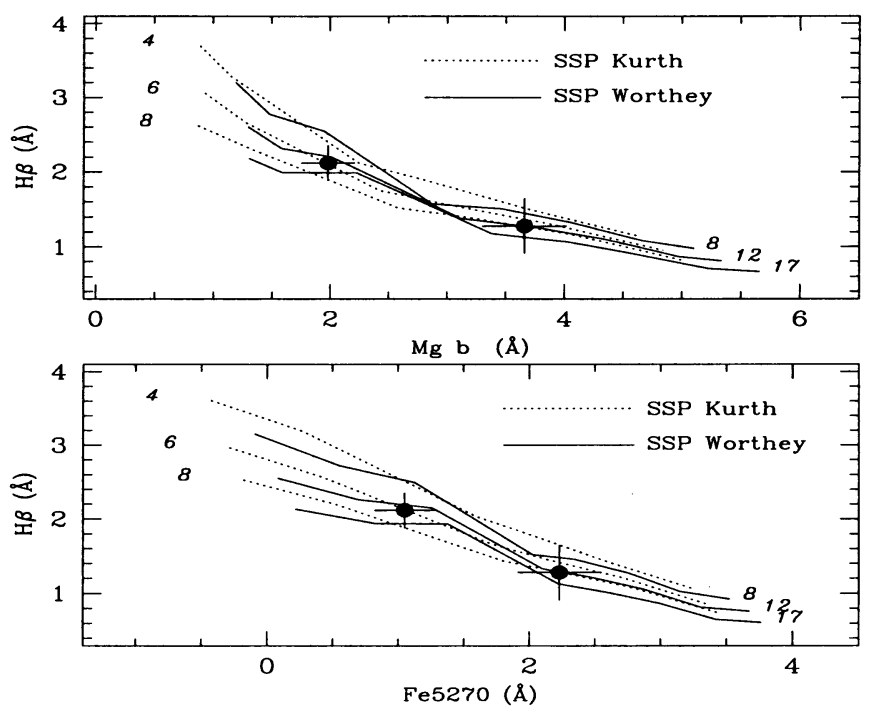

Figure 2. Ages of the red and blue GC sub-populations. Dotted lines represent SSP models of K99, solid lines are for those of W94. The isochrones of W94 indicate 8, 12 and $17 \mathrm{Gyr}$, those of KFF99 show 4, 6 and 8 Gyr.

blue and red GCs respectively. From KFF99, we obtain, $6.0 \pm 2$ and $8.0 \pm 5$ Gyr respectively. Therefore, we find that whilst the absolute ages between the models differ, there is no relative age difference between the blue and red GCs for either model. Within the uncertainties, the red and blue GCs are coeval.

\section{Acknowledgements}

M Beasley would like to thank his supervisor, Ray Sharples and his other collaborators involved in this project. M Beasley would also like to thank PPARC for its supporting studentship.

\section{References}

Beasley, M.A., Sharples, R.M., Bridges, T.J., Hanes, D.A., Zepf, S.E., Ashman, K.M., Geisler, D., MNRAS, 319, 1249

Davies, R.L., Sadler, E.M., Peletier, R.F., 1993, MNRAS, 262, 650

Geisler, D., Lee, M.G., Kim, E., 1996, AJ, 111, 152

Kurth, O.M., Fritze-v. Alvensleben, U., Fricke, K.J., 1999, AAPS, 138, 19

Worthey, G., 1994, ApJS, 95, 107

Zepf, S.E., Beasley, M.A., Bridges, T.J., Hanes, D.A., Sharples, R.M., Ashman, K.M., Geisler, D., 2000, AJ, 120, 2928 